\title{
CONCURRENT MALAKOPLAKIA OF CERVICAL LYMPH NODES AND PROSTATIC ADENOCARCINOMA WITH BONY METASTA- SIS: CASE REPORT
}

\author{
*A.L. GIDWANI, S.A. GIDWANI ${ }^{1}$, A. KHAN AND J.G. CARSON ${ }^{2}$ \\ Departments of General Surgery and ${ }^{1}$ Medicine, Causeway Hospitals Trust \\ Coleraine, Northern Ireland and ${ }^{2}$ Department of Histopathology, United Hospitals Trust, Antrim, \\ Northern Ireland
}

\begin{abstract}
SUMMARY
An unusual case of malakoplakia of the cervical lymph nodes in a patient with bony metastasis from prostrate cancer is reported. An 80-year-old patient with metastatic prostatic cancer presented with bilateral cervical lymphadenopathy, and a hard cervical mass in the left supraclavicular region. Biopsy of the lymph gland revealed the presence of malakoplakia, with no evidence of metastatic prostatic carcinoma. Though co-existence of malakoplakia and adenocarcinoma within the prostrate gland has been reported before, this case is unique, as it highlights the rare occurrence of malakoplakia involving distant nodes in a patient with bony metastatic prostate cancer. This report reflects the fact that things are not always what they seem in medicine, and not infrequently we come across a different pathology which mimics another.
\end{abstract}

Keywords: Malakoplakia, Lymph node, prostate, adenocarcinoma, Michaelis-Gutmann bodies

\section{INTRODUCTION}

Michaelis and Gutmann first described Malakoplakia in $1902^{1}$. It was only one year later that Von Hansemann named this disease as 'Malakoplakia' based on its gross appearances affecting the urinary bladder ${ }^{1}$. Though malakoplakia has been reported mainly to occur subsequent to infections, it could be associated with a variety of tumours ${ }^{2}$. By far, the commonest involvement of this inflammatory lesion is the genitourinary tract, mostly affecting the urinary bladder. Although coexistence of malakoplakia and adenocarcinoma within the prostate has been noted before ${ }^{3}$, this would be the first case ever reported with concurrent occurrence of malakoplakia of cervical lymph nodes in a patient with bony metastatic prostatic cancer.

\footnotetext{
${ }^{*}$ Author for correspondence
}

\section{CASE}

An 80-year-old male patient presented with fullness in the left side of the neck of few weeks duration. On examination there was bilateral cervical lymphadenopathy with a particularly hard lump in the right supraclavicular region clinically adherent to the sternomastoid muscle. One year prior to this presentation, he had a transurethral prostatic resection that showed adenocarcinoma. His recent investigations were suggestive of a metastatic prostatic cancer (PSA: $84.1 \mathrm{ng} / \mathrm{ml}$; Isotope bone scan: increased uptake throughout the skeleton, largest located in the lower pole of left scapula).

At surgery, a cervical lymph nodal mass, more than $5 \mathrm{~cm}$ in maximum dimension was identified which was matted and adherent to the left sternomastoid and surrounding structures. An excision biopsy of the suspicious central gland was performed. Histopathology revealed inflamed fibrous tissue with a granulomatous appearance. The inflammatory cells included lymphocytes, plasma cells and a conspicuous number of background histiocytes with eosinophilic cytoplasm. A significant number of histiocytes contained small calcified spherules, which were highlighted by a Von Kossa stain for calcium suggestive of MichaelisGuttmann bodies (Figure 1). There was no evidence of a metastatic tumour and an immunohistochemical stain for prostatic specific acid phosphatase was negative. Also stains for fungal organisms and acid-fast bacilli were negative. A histopathological diagnosis of Malakoplakia was made. A review of the prostatic chippings at transurethral resection of prostate (TURP) revealed a moderately well differentiated adenocarcinoma showing both focal cribriform pattern and ragged infiltration of fibromuscular stroma indicative of a Gleason grade 3+4 tumour (Figure2). 


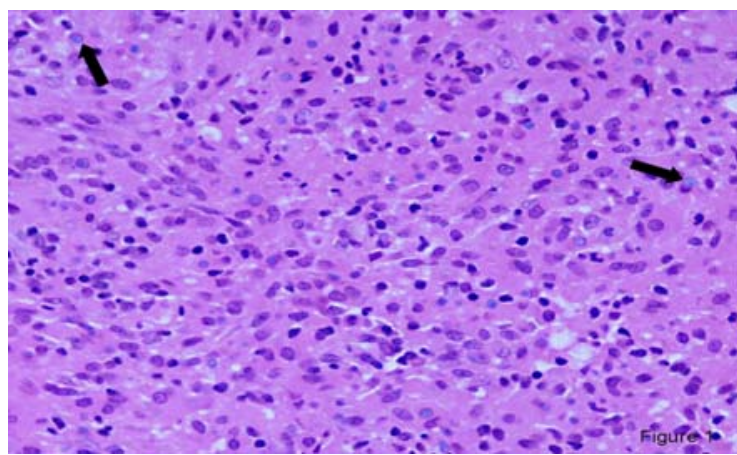

Figure 1 Lymph node biopsy: Background eosinophilic cytoplasm with histiocytes, few of which contain calcific spherules highlighted with Von Kossa stain suggestive of Michealis - Guttmann bodies with calcium

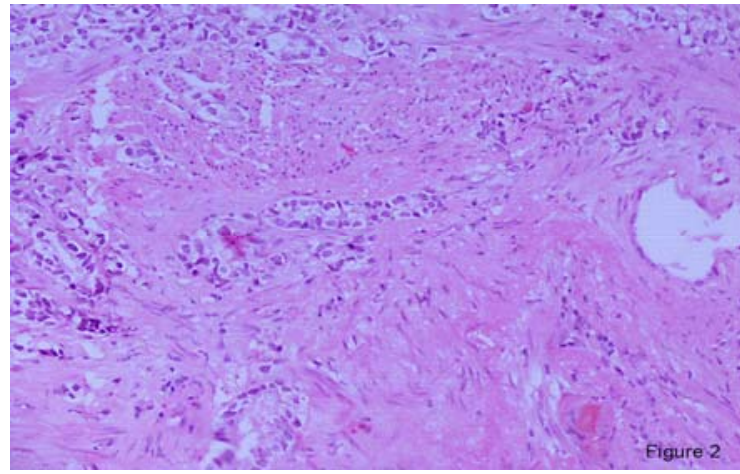

Figure 2 Prostatic biopsies (TURP): moderately differentiated adenocarcinoma with focal cribriform pattern and ragged infiltration of fibromuscular stroma indicative of Gleason grade 3+4.

While patient was commenced on hormonal treatment for the metastatic prostatic cancer, the malakoplakia was left untreated. Follow-up in the outpatient clinic revealed spontaneous regression in the size of the glands. The patient remains asymptomatic for more than four years with no recurrence of similar lumps.

\section{DISCUSSION}

Malakoplakia is a chronic inflammatory disease with disputed etiology and pathogenesis ${ }^{1}$. It is more common in middle-aged females (peak incidence in fifth decade compared with seventh in males $)^{4}$. It frequently affects the genitourinary tract though there have been reports where other sites as gastrointestinal tract, skin or lymph nodes have been involved. Though this condition is mostly associated with bacteria, viruses or parasites, the frequent occurrence with gram-negative bacterial infection as Escherichia coli and Proteus makes bacterial infection as a significant etiologic factor ${ }^{2}$. Also malakoplakia has been mostly known to af- fect the genitourinary tract where Escherichia coli is the commonest cause of infection. Since $E$. coli infection of genitourinary tract is very common and occurrence of malakoplakia is rare, there could be other patient factors as impaired immunologic function, which could modify the response to primary etiological agents.

The clinical features of malakoplakia are nonspecific and it can mimic a neoplastic process. Although malakoplakia has been associated with many types of malignancies, there has been no case suggesting malignant transformation of the condition $^{5}$. Histologically malakoplakia is considered as a granulomatous disorder. Ultra-structural examination reveals inflammatory infiltrate rich in plasma cells and histiocytes. These histiocytes differ from tumour cells in their uniform, round or oval vesicular nuclei and finely vacuolated cytoplasm $^{6}$. Within the cytoplasm of these histiocytes are found Michaelis-Guttmann bodies, which are characteristic to this condition. The bacteria in the macrophages stain positively with periodic acidschiff while the calcium in the MichaelisGuttaman bodies stain with Von Kossa².

The pathogenesis of malakoplakia is not clear. It occurs due to abnormal macrophage response to a primary cause, mostly an infection. This abnormal response is mostly secondary to impaired immunological function or chronic inflammatory process, which could explain the association of malakoplakia with conditions as Whipple disease, Chediak-Steinbrinck-Higashi syndrome, tuberculosis, sarcoidosis and immunedeficiency ${ }^{5}$. Malakoplakia, which very well mimics malignancy, carries a good prognosis and is self-limiting. In the present report, the authors hypothesise that an impaired macrophage response secondary to malignancy in the prostrate gland would explain the occurrence of malakoplakia in distant nodes.

Malakoplakia in the head and neck is very rare. A case of colonic and neck malakoplakia has been reported which responded very well to antibiotics and sulfathiazole ${ }^{1}$. Schmerber et al in 2003 reported a case of cervical malakoplakia, which clinically mimicked a cancer from hypopharyn $x^{5}$. The diagnosis was made at cervical biopsy and patient made satisfactory progress after commencing on antibiotics. In our case, the occurrence of malakoplakia of the neck nodes with no metastasis of tumour, in a patient with prostrate cancer with bony spread is probably incidental. Prostrate cancer usually spreads to pelvic nodes and metastasis to neck nodes is very rare. In our case, the diagno- 
sis of malakoplakia was made at biopsy and patient responded well to hormonal therapy. Four years have elapsed since presentation without any evidence of clinical recurrence.

\section{REFERENCES}

1. Chaudhry AP, Satchidanand SK, Anthone R, Baumler RA, Gaeta JF An unusual case of supraclavicular and colonic malakoplakia - A light and ultrastructural study. J Pathology 1980; 131: 193-208.

2. Darvishian F, Teichberg S, Meyersfield S, Urmacher CD Concurrent malakoplakia and papillary urothelial carcinoma of the urinary bladder. Annals of Clin \& Lab Sci 2001; 31: 147-150
3. Repassy DL, Ivanyl A, Csata S, Tamas G. Combined occurrence of prostate cancer and malakoplakia. Pathol Oncol Res 2002; 8(3): 202-203.

4. McClure J. Malakoplakia of the urinary tract. Br J Urol 1982 Apr; 54(2): 181-5.

5. Schmerber S, Lantuejoul S, Laviellie JP, Reyt E Malakoplakia of the neck Arch Otolaryngol Head Neck Surg 2003; 129: 1240-1242.

6. Agostinho AD, Correa LA, Amaro JL, Bacchi CE, Camargo JLVD Malakoplakia or prostate cancer? Similarities and differences Urol Int 1998; 61: 47-49 